\title{
Flax (Linum usitatissimum L.) response to Fusarium oxysporum infection on transcriptome level
}

\author{
Novakovskiy R.O. ${ }^{1 *}$, Krasnov G.S. ${ }^{1}$, Rozhmina T.A. ${ }^{1,2}$, Pushkova E.N. ${ }^{1}$, \\ Povkhova L.V..$^{1,3}$, Kezimana P. ${ }^{1,4}$, Kudryavtseva L.P. ${ }^{2}$, Dmitriev A.A. ${ }^{1}$, Melnikova N.V. ${ }^{1}$ \\ ${ }^{1}$ Engelhardt Institute of Molecular Biology, RAS, Moscow, Russia \\ ${ }^{2}$ Federal Research Center for Bast Fiber Crops, Torzhok, Russia \\ ${ }^{3}$ Moscow Institute of Physics and Technology, Dolgoprudny, Russia \\ ${ }^{4}$ Peoples' Friendship University of Russia (RUDN University), Moscow, Russia \\ *e-mail:0legovich46@mail.ru
}

Flax (Linum usitatissimum L.) is used for production of textile, vegetable oil, composite materials, and pharmaceuticals. Fusarium wilt caused by the fungus Fusarium oxysporum sp. lini is the most harmful pathogen that reduces flax production. For obtaining high yields of flax, cultivation of cultivars that are resistant to $F$. oxysporum is necessary. Methods of classical genetics revealed different genes for resistance to $F$. oxysporum, however, sequences and products of these genes are still unknown. In the present study, susceptible and resistant to Fusarium wilt flax cultivars and lines, including those that according to classical genetic analysis carry different resistance genes, were studied. Transcriptome sequencing was performed for susceptible and resistant genotypes grown under control conditions and 48 hours after inoculation with $F$. oxysporum spores. About 250 million reads were generated on NextSeq Illumina. After trimming of the reads using Trimmomatic and filtering against the F. oxysporum genome, the flax transcriptome assembly and annotation were performed. Then reads were mapped to the assembled transcripts and quantified using bowtie 2 and rsem. The scoring was developed to identify genes with diverse expression alterations in resistant and susceptible to $F$. oxysporum genotypes, and resistance gene candidates were identified. Our study contributes to the understanding of the key mechanisms of flax response to F. oxysporum, identification of resistance genes, and development of molecular markers for breeding of flax cultivars carrying several resistance genes simultaneously.

Acknowledgements: This study was supported by the Russian Science Foundation. This work was financially supported by the Russian Science Foundation, grant 16-16-00114. 